\title{
DOMAINES FOURNISSEURS DES EXPRESSIONS FIGÉES
}

Danijela Ljepavić, Université du Monténégro, danijelalj@t-com.me

10.31902/fll.29.2019.13

UDK 81.163.4'374:81.133.1'374

UDK 82.035

Résumé: L'origine des expressions figées est de natures diverses. Les unes sont des « façons de parler » qui ont fait fortune parce qu'elles ont plu par leur caractère expressif, sans qu'on puisse dire si c'est une image, ou une comparaison amorcée ou expérimentée, qui leur a valu ce succès. Cependant, à l'origine toutes les expressions figées ont été motivées : comparaison, métaphore, images ou clichés, certaines ont totalement perdu leur sens premier, d'autres s'adressent encore à l'imagination du locuteur et suggèrent un rapport avec leur étymologie.

Mots clés: origine, étymologie, expression, figement, traduction.

\section{Introduction}

Cet article montre que l'histoire n'appartient pas qu'aux historiens. C'est ainsi que la mémoire collective s'approprie des événements ou des personnages sous forme d'expression ou de citations qui les caractérisent et gravent un fait dans la mémoire, en entrant dans le langage courant.

Parfois, une expression paraît si bien caractériser un événement ou un personnage qu'elle lui est devenue indissociable : que seraient Colomb sans son œuf ou Louis XIV sans son « l'État, c'est moi » ? D’autre fois, la référence historique est complètement oubliée sans que l'expression elle-même disparaisse : le Gravelotte du « ça tombe comme à Gravelotte » ne doit pas dire grand-chose à la plupart d'entre nous.

Dans cet article nous avons fait un choix des expressions figées de différents domaines fournisseurs : histoire, croyances et superstitions, religion et temps. Nous n'avons retenu que celles qui sont encore régulièrement employées.

\section{Les origines historiques des expressions figées}

Les gens perçoivent le monde qui les entoure. Ils forment une vision collective de tout ce qui se passe autour d'eux et ils en parlent en utilisant les expressions imagées surtout quand il faut décrire une chose qui n'est pas matérielle et qui a une autre dimension. C'est pour cela qu'il est utile de connaître les stéréotypes, les croyances, les superstitions et les coutumes d'un peuple pour mieux comprendre leur impact dans la langue. 
Il est dans la nature des locutions de retenir leur motivation, car les mots qui les composent, bien que formant une unité, gardent une certaine autonomie et continuent à évoquer des images qui leur sont propres. Ainsi le sens est clairement motivé dans tenir le gouvernail, tondre un œuf, lever un lièvre; mais dans la plupart des cas, l'image libérée par la locution s'actualise sans révéler le lien sémantique qui est à la base des valeurs particulières de l'expression.

\section{Domaines fournisseurs}

L'objectif de cet article est de donner l'origine des expressions BCMS (bosniaque/croate/monténégrin/serbe) plus que des expressions françaises, car il y a plus d'ouvrages en français qu'en BCMS qui traitent cette problématique. Nous pouvons constater que l'histoire, la religion mais aussi des croyances et des superstitions jouent un grand rôle dans les expressions figées.

Nous avons donné seulement des exemples pour quelques domaines fournisseurs et il s'agit de notre choix d'exemples à partir des ouvrages de Milan Šipka, Dragana Mršević Radović et de Veselin Čajkanović.

\subsection{Histoire}

L’histoire nous a légué de nombreuses expressions en BCMS :

Dati svoj obol

Traduction littérale et équivalent français : apporter son obole

L'histoire vient de la Grèce parce que l'obole était une unité monétaire qui a eu cours dans la Grèce antique. L'obole (en grec ancien ỏßchóc / obelós et en attique óboגós / obolós, d'abord " broche ») est une monnaie antique valant un sixième de drachme. Dans l'étalon monétaire athénien, l'obole pèse 0,72 grammes. Elle est divisée en huit chalques. Elle est d'abord frappée en argent puis, à partir de l'époque hellénistique, en bronze.

Avant d'entrer dans l'expression, le mot obole avait une valeur religieuse. Selon le rite funéraire grec, on devait placer dans la bouche des morts une obole pour leur permettre de payer à Charon le passage du Styx. Ceux qui ne pouvaient pas payer étaient condamnés à errer sur les berges du Styx durant cent ans. Les anciens grecs donnaient ainsi une obole pour aider les morts pour passer dans l'autre monde c'est-à-dire dans l'éternité.

À partir du XVIle siècle, comme le denier, l'obole avait connu également une dévaluation continuelle tout au long du Moyen Âge, le mot fut employé au sens de "somme d'argent minime ", puis, à partir du XIXe siècle, au sens de " présent de peu de valeur ». Comme la valeur de l'obole n'était pas grande, 
tout le monde pouvait la donner : soit à Charon pour le passage, soit en tant qu'aumône. II vient de cela qu'on dit donner son obole pour dire donner un peu d'argent en tant qu'aumône à quelqu'un ou donner quelque chose pour l'aider. C'est aussi un sens symbolique: " faire un (beau) geste pour aider quelqu'un ».

\section{Isti aršin / Na istoj deredži}

Traduction littérale : même mesure

Signification et équivalent en français : juger/mesurer à sa toise

Il est très fréquent d'avoir des mots turcs ou perses dans la langue BCMS, le territoire où l'Empire ottoman régna pendant cinq siècles. Dans l'expression isti aršin, le mot aršin vient du tucs (arşin) et veut dire la mesure de coudée.

Cette mesure se situait entre 65 et 75 centimètres, et était toujours différente dans différents pays. Avec l'autre expression na istoj deredži, avec la même signification, on se rend compte que les expressions seraient en effet transparentes si l'on connaissait les mots turcs aršin et deredža. Ces deux mots avaient été largement utilisés à l'époque de l'Empire ottoman mais aujourd'hui on ne les utilise que dans ces expressions, d'où leur opacité.

\section{Španska sela}

Traduction littérale : les villages espagnols

Signification et équivalent en français : c'est du chinois

En BCMS quand quelque chose nous est inconnu, incompréhensible ou très flou, on dit que c'est des villages espagnols pour nous. On peut se demander pourquoi espagnols, pourquoi pas allemands ou portugais, par exemple ? Et surtout pourquoi le pluriel "des villages » ? II est curieux aussi qu'on utilise les villages et non pas la forêt ou la jungle, par exemple.

Pour expliquer cette expression on aura recours au dictionnaire allemand-serbo-croate par Pavica Mrazović et Ružica Primorac (1981) et aux notes d'Ivan Klajn.

Tout d'abord il faut dire qu'il s'agit de la traduction littérale de l'expression allemande spanische Dorfer (villages espagnols) avec la même signification qu'en BCMS. Karlo Brudor affirme que cette expression a été utilisée pour la première fois par Johann Wolfgang von Goethe dans son roman "Les Souffrance du jeune Werther ». Traduit en serbe (Branko Mušicki, Mala biblioteka IIIV, Mostar 1905) la phrase contenant l'expression dont on parle est la suivante : "To su bila za poslanikov mozak španska sela, i ja se preporučim da se ne bih morao još više žučiti zbog daljeg nerazumnog govora. " (Pour le cerveau du 
député ce n'étaient que des villages espagnols et je me recommande afin de ne plus me fâcher à cause de ce discours incompréhensible).

Il est possible que grâce à cette phrase du roman, la langue BCMS ait adopté cette expression qu'on a commencé à utiliser dans la vie quotidienne.

Il est curieux que l'expression « španska sela - spanische Dörfer » a prévalu sur « bömische Dörfer - les villages tchèques ». En allemand, « bömische Dörfer » existe depuis la guerre de Trente ans. Les soldats allemands en traversant le pays Tchèque avaient du mal à retenir les noms slaves qui leur paraissaient bizarres et par conséquent tout ce qui était incompréhensible ils l'appelaient « les villages tchèques »-bömische Dörfer).

Ce phénomène existe aussi dans les autres langues, comme par exemple en espagnol « esto es griego para mi - c'est du grec pour moi ». En anglais pareil " It's Greek to me ». Les Espagnols disent aussi « esto es arabigo para mi - c'est de l'arabe et les français disent " c'est du chinois " ou " c'est de l'hébreu ".

On peut conclure que la langue qui n'était pas parlée dans un pays devenait facilement très bizarre et incompréhensible, d'où vient l'explication de ces expressions. L'espagnol était incompréhensible pour les Allemands, le grec et l'arabe pour les Espagnols et ainsi de suite. La langue chinoise étant peu connue en Europe occidentale et représentant un type linguistique absolument étranger (surtout à cause de son écriture non alphabétique, non latine), il était normal qu'elle serve de symbole de l'extrême difficulté de compréhension, d'où vient « c'est du chinois » chez les français. Pour le BCMS, c'était le calque direct de l'allemand qui nous a légué l'expression « les villages espagnols ».

\section{Nositi glavu u torbi / Staviti glavu u torbu}

Traduction littérale : porter/mettre sa tête dans le sac

Signification et équivalent en français : risquer sa peau ; marcher sur le fil du rasoir

Staviti glavu u torbu " mettre la tête dans le sac ", veut dire : s'exposer à un danger/ risquer sa vie. Au cours de l'histoire mais aussi de nos jours, les gens " mettent leur tête dans le sac » soit s'il s'agit d'aller à la guerre soit d'exercer une profession dangereuse (les acrobates, les parachutistes, etc.).

L'origine de cette expression dans notre langue vient de l'histoire de la guerre. Au Moyen Age, et même avant, on connaît la coutume de couper la tête des ennemis et de la montrer aux souverains comme preuve de la bataille gagnée. La Bible nous dit que la même coutume existait lors de la bataille où David a vaincu le géant Goliath. Il est ainsi écrit : « Et David prit la tête du Philistin et l'apporta à Jérusalem, et il mit son armure dans sa tente. "

Le plus ancien souvenir de cette coutume guerrière chez nous a été noté dans « La vie de Milutin Nemanjić » par l'archevêque Danilo II (1970). 
"Ils prirent le commandement ennemi, Crnoglava-Tête-noire, ils lui coupèrent la tête et la mirent sur la lance pour l'apporter au roi Milutin en tant que cadeau spécial ».

Cette coutume des têtes coupées a été gardée jusqu'au milieu du XIX siècle. L'histoire note que le prince Miloš Obrenović avait envoyé à Constantinople en 1817 la tête du leader du premier soulèvement serbe, Karađorđe Petrović. Les têtes de Turcs avaient été apportées aussi à l'évêque Petar Petrović Njegoš avec lesquelles ils décoraient Cetinje (ancienne ville royale du Monténégro). La plus appréciée était la tête de Smail-aga Čengić. Smail-aga Čengić était un bey bosniaque ottoman et le général de l'armée ottomane, qui a lutté contre les insurgés serbes entre 1809 et 1813, pendant la Première insurrection serbe, dirigée par Đorđe Petrović. Au début des années 1830, Čengić lutta contre le dragon de la Bosnie, Husein Gradaščević, qui menait une rébellion contre le gouvernement bosniaque central ottoman pendant la Grande révolte de Bosnie. Čengić a été tué par le bandit serbe Novica Cerović en se vengeant pour avoir tué le frère cadet du prince-évêque du Monténégro, Petar II Petrović Njegoš. Sa mort a inspiré en 1846 le poème épique La mort de Čengić Smail-aga écrit par Ivan Mažuranić.

Ces têtes avaient été portées dans un sac ou dans une musette, d'où vient l'origine de l'expression mettre la tête dans le sac pour dire être en danger/ risquer sa peau.

\section{S koca i konopca / kusi i repati}

Traduction littérale : des gens de sac et de corde/ceux avec et sans la queue

Signification et équivalent en français : des gens sans foi ni loi, des gens de sac et de corde

En français la même expression, des gens de sac et de corde est une allusion claire au traitement des malfaiteurs qui pouvaient être enfermés dans un sac noué d'une corde et noyés, ou pendus avec une corde.

On trouve cette expression très utilisée chez des écrivains très connus tels que : Vuk Karadžić, Rodoljub Čolaković, Baltazar Bogišić. On I'utilise quand on veut dire qu'un groupe est composé de personnes de toutes sortes et surtout de mauvaises.

Pourquoi le dit-on?

Il est connu qu'au XIXe siècle, surtout pendant l'Empire ottoman, les gens qui avaient commis un délit ou avaient fait quelque chose de mal, étaient condamnés à mort. Ils étaient empalés ou pendus. Il y en avait qui se sont enfuis et qui sont en effet les gens du pal et de la corde. 
Ivo Andrić décrit dans son roman Na Drini ćuprija (Le pont sur la Drina) le processus de l'empalement et de la pendaison. Il y a d'autres expressions inspirées par ceux-ci qui veulent dire : être empalé - omastiti kolac (engraisser le pal) ; omastiti konopac (engraisser la corde) - être pendu ; sukati kome konopac (faire la corde à quelqu'un) - lui préparer la mort ; goniti kocem i konopcem (chasser par le pal et la corde) - punir impitoyablement.

\section{Biti na bijelom hljebu}

Traduction littérale : être au pain blanc/manger du pain blanc

Equivalent français : manger son pain blanc

Les pauvres ne pouvant pas se permettre acheter du pain blanc ils se contentent de manger du pain noir ou demi-blanc. Ce fait pourrait nous faire penser que l'expression manger du pain blanc veut dire être riche et mener une vie somptueuse. Mais ce n'est pas vrai.

Il est intéressant qu'en BCMS l'explication de l'expression manger du pain blanc, veut dire attendre l'exécution de la peine de mort ou être en attente de la résolution d'une situation, d'un problème. Dans ce genre de situation on dit souvent qu'on ne sait pas quel destin nous attend. Mais en français, le sens est différent et s'oppose à l'expression manger du pain noir. La construction française serait plutôt : manger son pain blanc en premier. On plaint la personne de mal vivre suite à un destin ou des circonstances de vie difficiles. La signification est : commencer par des choses agréables, réussir d'abord avant de subir des désagréments. L'explication de cette expression en BCMS vient de la jurisprudence au temps où la peine de mort existait. Les prisonniers dans cette situation étaient nourris de pain blanc et de la meilleure nourriture puisqu'ils vivaient leurs derniers jours. L'ethnologue Veselin Čajkanović (1955) explique que la coutume de la préparation d'une nourriture délicieuse et riche pour les condamnés à mort fait partie de la tradition d'antan.

\section{Bacati drvlje i kamenje}

Traduction littérale : jeter du bois et des pierres.

Il est difficile de trouver l'équivalent en français. II n'y a pas d'expression figée avec la même signification. On peut seulement essayer d'expliquer en utilisant une paraphrase : répandre des calomnies sur quelqu'un ; dire peste et rouge de quelqu'un; se répandre en invectives.

L'expression trouve son origine dans une coutume très ancienne qui date du Moyen Age chez les Egyptiens, Persans, Juifs, Grecs, Romains, Gaulois et les 
autres anciens peuples avant l'exécution de la peine de mort. Le cheminement vers le lieu d'exécution et l'exécution elle-même sont l'objet d'une cérémonie dont tous les moments ont un sens. La rue conduit au supplice, dans chaque ville, toujours la même et elle doit être peuplée par un abondant public. La foule peut être sollicitée lors des arrêts du cortège, en général aux carrefours, pour insulter le condamné ou lui jeter des pierres et de la boue. « Battez fort et n'épargnez point ce paillard, car il a bien pis desservi ! ", crie encore le public sous le règne de Louis XI. Le condamné est mis dans une charrette, la charrette d'infamie qui est aussi celle de la boue des rues et des ordures. Au moment de l'exécution, un responsable de la justice crie l'acte d'accusation, le dictum, au peuple qui l'écoute. Puis le bourreau fait son office.

Le seul acte de chasser le condamné du village signifie qu'on l'expulse de la société où il a commis son crime. L'endroit de l'exécution où la foule jetait des pierres et du bois jusqu'à la mort du condamné était considéré comme maléfique.

Les gestes et les cris qui scandent ces cérémonies ont un sens symbolique. La peine doit prendre une valeur exemplaire et le pouvoir affirme ainsi sa force. II manifeste aux yeux de tous qu'il a le pouvoir de vie et de mort. La foule est donc là pour prendre exemple et, parce qu'elle est terrorisée, pour être dominée. Mais la foule est aussi là pour participer à l'exécution et sa présence est nécessaire à l'accomplissement de la peine. Elle est témoin de l'infamie qui, peu à peu, au cours du rituel judiciaire, rejette le condamné hors du monde des vivants, l'exclut comme inutile au monde et irrécupérable.

\section{Kom opanci, kom obojci}

Traduction littérale : I’un obtiendra opanci, l'autre obojci

Signification et équivalent français : advienne que pourra

Pour comprendre cette expression, il faut d’abord connaître la signification des mots opanak/pl. opanci et obojak/pl. obojci.

L'Opanak est une sorte de chaussure légère que l'on lace autour de la cheville. Les opanci serbes sont très connus sous le nom šiljkani. Le mot obojak ou obojci au pluriel n'est pas très connu parce que ça fait longtemps qu'on ne les porte plus. Les Obojci se portaient comme les chaussettes aujourd'hui. II s'agissait d'une étoffe dans laquelle on enroulait les pieds pour pouvoir porter les opanci sans qu'ils fassent des cals.

L'expression kom opanci, kom obojci - signification : " l'un obtiendra opanci et l'autre obojci » après une bataille/dispute, fait partie des expressions qu'on utilise fréquemment dans la langue BCMS sans pour autant en connaître l'origine. 
Vuk Stefanović Karadžić ( 1956) explique dans le « Dictionnaire des proverbes serbes " qu'il est mieux d'avoir des opanci (chaussures) que des obojci (chaussettes) qui seuls ne nous servent à rien. II est aussi possible que si quelqu'un reste dans les opanci, il reste vivant par rapport à celui qui garde souvent les obojci après avoir perdu ses opanci lors d'une bataille. Alors, l'expression veut dire : on va entrer en bataille et on verra qui est plus fort ou bien qui restera dans les opanci (dans les chaussures) et qui restera dans les obojci (dans les chaussettes).

\section{Imati putra na glavi}

Traduction littérale : avoir du beurre sur la tête

Signification et équivalent français : avoir un cadavre dans le placard ; avoir mauvaise conscience; avoir un poids sur la conscience

Cette expression est très utilisée notamment en Voïvodine et dans la partie centrale de la Serbie. D’après Pavica Mrazović et Ruzica Primorac (1981), auteurs du dictionnaire phraséologique allemand/ serbo-croate, cette expression est la traduction de l'expression allemande : Butter auf dem Kopf haben. Ils expliquent aussi que cela provient du proverbe Wer Butter auf dem Kopf hat, der soll nicht in die Sonne gehen (celui qui porte du beurre sur la tête, ne doit pas s'exposer au Soleil).

Une situation réelle en Allemagne et en Serbie peut expliquer ce proverbe et ainsi l'expression. A l'époque il fallait payer des impôts sur les produits importés des villages dans les villes. Le beurre était très cher et les femmes le portaient dans des paniers sur la tête pour le cacher en passant vers la ville. Donc elles avaient vraiment du beurre sur la tête et il ne fallait surtout pas s'exposer au soleil pour ne pas le faire fondre et ainsi se dévoiler.

En français, c'est plutôt une métaphore analogue au skeleton in the closet de l'anglais qui a donné l'expression avoir un cadavre dans le placard.

\subsection{Croyances et superstitions}

L'aller et retour entre discours individualisé et codage social s'observe parfaitement dans cet univers mouvant des expressions figées. Son évolution est fonction des systèmes de communication, qu'ils soient interindividuels ou massifs c'est-à-dire des mouvements culturels de la société toute entière.

Aleksandar Belić (1951) explique que la formation des mots dans une langue dépend des circonstances dans lesquelles une société vit, de la nature qui l'entoure, de la culture qui la nourrit. De ce fait, le contenu des mots d'un peuple d'une civilisation et culture donnée diffère d'un autre peuple. Belić sou- 
ligne ici que pour la formation des pensées, les coutumes, les habitudes et les croyances jouent un grand rôle.

\section{Tjerati kera}

Traduction littérale : chasser le chien

Signification et équivalent français : faire la bombe ; faire la noce/la foire/la bringue

L'expression tjerati kera est une vraie expression figée opaque parce que la phrase elle-même ne nous incite guère à penser qu'il s'agit d'une foire ou d'une fête. Le mot ker veut dire un chien et pour dire la même chose, il existe aussi le verbe qui vient de ce mot : keriti se. La signification serait la même : faire la noce/la foire.

Velimir Mihailović, professeur à la Faculté de philosophie à Novi Sad, a essayé d'expliquer l'origine de cette expression. Il a utilisé l'article Deux fêtes du culte animal de Veselin Čajkanović, ethnologue serbe, et il explique que l'origine du mot ker (chien en BCMS) a une histoire païenne. Il s'agit des fêtes de chiens. La plupart des fêtes, qui comprennent la débauche et le libertinage, finissent par la chasse du mal. En bulgare il existe une expression « kučeški ponedelnik (lundi de chien) » qui signifie lundi pur (première semaine après le jeûne). La fin de la foire (la chasse du mal) se manifeste en chassant le chien pour cette année. Le rite de l'adoration du chien et de sa liquidation à la fin de la fête a fait naître l'expression terati kera. Cet acte est fait avec l'ivresse et le débordement comme les antiques bacchanales d'où vient l'explication de faire la fête quand on pense à chasser le chien. La même expression existe en roumain et en russe et d'après le professeur Mihailović, le mot ker (de l'origine care) est d'origine roumaine (Šipka 2008).

\section{Pasti s kruške}

Traduction littérale : tomber du poirier

Signification en français : être considéré comme un fou

Equivalent français : je ne suis pas né de la dernière pluie

Dans la mythologie slave, l'on trouve les croyances spécifiques pour concernant certaines plantes comme par exemple le poirier qui était l'arbre pour les démons et pour les fées. C'était leur temple et si quelqu'un voulait monter sur cet arbre, les fées et les sorcières le poussaient pour qu'il tombe et il en devenait forcément fou, d'où vient l'expression je ne suis pas tombé 
$d u$ poirier. On utilise cette expression aussi comme adjectif pour dire kao da je pao s kruške (comme s'il était tombé du poirier) pour décrire quelqu'un qui est confus, distrait. Dans le dictionnaire "Matica srpska » on trouve aussi l'idiome " kao s kruške » (comme du poirier) pour dire répondre d'une manière grossière/rude.

On se demande pourquoi les mots qui décrivent les caractères négatifs sont associés au poirier. Pour expliquer la signification culturelle du poirier on va avoir recours au livre de Veselin Čajkanović (1985) « Dictionnaire des croyances serbes des plantes ». A la différence du pommier, Čajkanović explique que le poirier est considéré comme l'arbre du mal, l'arbre des démons. D’après les croyances, les diables se cachent sous le poirier et les sorcières s'installent sur le poirier. En Slavonie, la croyance dit que les sorcières utilisent de la graisse de la poire pour s'envoler.

Puisque cet arbre s'appelle l'arbre du mal, il est facile de comprendre que les gens considéraient fous, distraits et ensorcelés ceux qui se mettaient sur le poirier et en tombaient ensuite. On constate que la réponse du diable ou de la sorcière ne peut être que rude, voici comme on explique l'idiome répondre comme du poirier (odgovoriti kao s kruške).

\section{Udariti glogov kolac}

Traduction littérale: enfoncer le bâton d'aubépine

Signification et équivalent en français : donner/porter le coup de grâce, le coup mortel

Cette expression trouve son origine dans les croyances des vampires et des loups-garous. Vuk Stefanović Karadžić, dans le "Dictionnaire serbe " explique que Vukodlak (loup-garou) est l'homme dans lequel entre l'esprit du diable 40 jours après sa mort et le rend vivant. Ainsi, le loup-garou sort-il de la tombe dans la nuit et étrangle les gens dans les maisons. D’après la croyance, l'homme probe ne devient jamais un vampire sauf si un animal le survole. C'est pourquoi on veille à ce que rien ne survole le cadavre.

Le loup-garou sort de la tombe en hiver et quand le nombre de gens morts dans un village augmente on dit qu'il y a un mort qui est devenu le vampire. Ne sachant quel tombeau cache le vampire les gens prennent un corbeau et le mettent au-dessus des tombeaux dans lesquels le vampire pourrait se cacher. On croit que le jeune corbeau ne passe jamais au-dessus du tombeau où se trouve le vampire. S'ils trouvent qui pourrait être le vampire, ils exhument le cadavre et s'il n'est pas en état de putréfaction, ils le piquent avec les bâtons d'aubépine et le mettent dans le feu. Le loup-garou trouvé dans le tombeau est, d'après la légende, toujours gros et rouge du sang pris aux gens. Cette histoire 
racontée ainsi par Vuk Stefanović Karadžić témoigne que notre peuple croyait que c'était seulement le bâton de l'aubépine qui pouvait donner le coup mortel et détruire un mal.

Čajkanović (1955) dit que les gens racontaient qu'il suffisait d'une épine pour faire sortir le diable. C'est pourquoi on pique le cadavre avec l'épine de l'aubépine sur le ventre avant de le mettre dans la tombe. La branche de l'aubépine se met derrière le cadavre un jour avant l'enterrement. Quand il n'est pas évident de savoir dans quelle tombe se trouve le vampire, les gens mettent les bâtons dans toutes les nouvelles tombes pour s'assurer que le cadavre ne devienne pas le vampire. Et pour assurer que le vampire n'entre pas dans la maison, les gens mettent les bâtons sur les fenêtres et sur les portes.

Que quelqu'un mentionne le vampire et tout le monde dit : na putu mu broć i trnje (que sur son chemin se trouve la garance et l'aubépine). C'est ainsi que d'après la croyance l'aubépine est devenue le remède contre le mal, les vampires, les sorcières et la maladie. On la met aussi comme talisman qui garde notamment les enfants contre les vampires et le mal. La religion slave ne s'est pas partout développée au point de former un panthéon unique, tandis que la démonologie était la propriété commune. On considère que toutes nos croyances dans les démons se sont maintenues plus longtemps puisque l’Église les avait négligées dans sa tendance à effacer le plus tôt possible la mémoire des divinités païennes.

\subsection{Religion}

L'église, son droit, sa liturgie, ses coutumes sont de même à l'origine de nombreuses expressions qui s'expliquent clairement à la lumière de leur contexte historique et qui ont survécu dans des métaphores.

En français :

Avoir droit au chapitre

Etre à la portion congrue

Jeter son froc aux orties

Donner de l'encensoir

Sentir le fagot

Mettre à l'index

Ne savoir à quel saint se vouer (calque)

\section{En BCMS :}

avoir le droit de donner son avis - pravo na mišljenje avoir un revenu juste suffisant pour vivre - imati dovoljno se libérer d'une contrainte - osloboditi se muke faire des compliments outrés -pretjerati u komplimentima être soupçonné d'hérésie - optužen za jeres exclure d'une compagnie - isključen iz grupe ne savoir à qui recourir - ne znati kojem svecu da se moli

Certaines de ces expressions ne sont compréhensibles qu'en connaissance d'usage, de faits particuliers ou tombés en désuétude. Pa exemple en français : la rubrique dans l'expression savoir la rubrique désigne le texte en 
lettres rouges qui indique dans les missels la manière de célébrer ou de suivre l'office. C'est donc « connaître la pratique d'un métier ». On ne trouve pas l'expression équivalente en BCMS.

Les situations, les personnages, les paraboles et les métaphores bibliques sont les sources de nombreuses locutions.

Certaines expressions réfèrent à des traits de mœurs des anciens Hébreux. Il s'agit très souvent des calques dans les autres langues y compris BCMS :

Le bouc émissaire : " personne sur laquelle on fait retomber les fautes communes ", c'est une allusion à la fête des expiations au cours de laquelle un bouc symboliquement chargé des péchés d'Israël était envoyé dans le désert.

En BCMS : Žrtveni jarac

Adorer le veau d'or: " faire bassement sa cour aux riches et aux puissants ", rappelle la conduite d'Israël dans le désert aux temps où il était à la recherche de la terre promise par Dieu à son peuple et vers laquelle Moise devait le conduire. L'expression le veau d'or apparaît en français avant même les premières traductions de la Bible. En BCMS : Klanjati se novcu.

On a tiré surtout un grand nombre d'expressions du Nouveau Testament. Les unes sont des allusions à la vie et à la Passion du Christ.

Porter sa croix « être sujet à des peines journalières ». En BCMS : Nositi svoj krst. S'en laver les mains "dégager sa responsabilité ", comme le fit Ponce Pilate après la condamnation de Jésus par les Juifs. En BCMS : calque : Oprati ruke.

La couronne d'épine "souffrances imméritées et subies par les justes et les innocents ». En BCMS : nepravedna patnja/Isusove muke - les peines de Jésus Christ.

D’après la Bible, Dieu créa Adam (adamah- terre) et on dit que Dieu était premier potier puisque il a créé Adam de la terre. Et Adam était potier aussi puisque il a fait un pot pour manger des lentilles. II vient de cela une expression en BCMS otišao da pravi lonce " il est parti pour faire les pots » et cela signifie qu'il est mort. L'expression s'explique par l'image que l'homme rentre dans la terre de laquelle il était né et il peut en faire des pots.

Une autre expression peut être liée à cette histoire. C'est pravićeš me od blata " tu me feras de la boue ", tu vas me regretter. Donc, cette personne nous manquera tellement que nous allons vouloir le faire de la terre/de la boue comme Dieu a fait Adam. Mais tout cela sera en vain parce qu'il n'y a qu'un seul Dieu et personne ne peut faire un être humain sauf lui.

II y a aussi l'expression bogu iza leđa " derrière le dos de Dieu " pour dire très lointain, un endroit inconnu même de Dieu. En français nous avons l'expression à Trifouillis-les-oies et en BCMS on invente un lieu comme il est parti à Prdina bara ou aller à Piskopeja ce qui est un petit village en Albanie. Les 
consonnes « sk » jouent un rôle important parce qu'elles donnent la tonalité à l'expression.

En général, tout espace qui ne nous est pas connu appartient aux démons, aux vampires et Dieu n'y est pas présent. C'est pour cela que nous avons encore une expression pour dire la même chose gdje je Bog rekao laku noć" le lieu où même le Dieu a dit bonne nuit ".

Quand on veut dire que quelqu'un ou quelque chose a disparu sans qu'on sache où il se trouve il y a une expression en BCMS kao da ga je zemlja progutala " comme si la terre l'avait avalé ». Cela vient de la croyance que la terre a des trous dans lesquels on tombe dans un autre monde, le plus souvent le monde des morts et des démons. De là vient l'expression otići bestraga « partir sans laisser la trace " qui veut dire disparaitre aux yeux de quelqu'un mais cela ne veut pas dire que quelqu'un est mort ! "Bestrag " signifie quelque chose qu'on ne peut pas concevoir ni toucher, ni en savoir plus. Cet espace lointain est toujours réservé aux démons et vampires.

La peur était et est toujours restée un domaine très riche pour la phraséologie. Depuis que l'homme existe il a peur de quelque chose. Avant la religion c'était des démons et des sorcières, ainsi que d'autres créatures divines. Avec la religion c'est le diable qui remplace tous les maux. Chaque peuple slave avait ses propres armes contre ces créatures qui font du mal aux gens comme accrocher les gousses d'ails à la porte d'entrée ou la sorcière en paille contre la mauvaise volonté de ceux qui nous rendent visite. La croix est un symbole très porté et gardé dans la maison de tous les slaves orthodoxes et on dit souvent bježi kao đavo od krsta " fuir comme un diable devant la croix/s'agiter comme un diable dans un bénitier ". L'encens est aussi même aujourd'hui conçu comme un porte-bonheur qui chasse tous les maux.

Même pour dire que quelqu'un est laid il y a beaucoup de comparaisons. Laid comme un singe, un voleur, un minuit, un cimetière, etc. L'équivalent en français pour quelqu'un qui est très laid serait qu'il est un vilain magot. Pourquoi utilise-t-on l'étalon singe pour dire que quelqu'un est laid et malin ? D'abord parce que dans la religion chrétienne et surtout dans l'iconographie, le singe est décrit comme un homme mais qui a plein de vices, qui est hypocrite, malin et rusé. L'homme a évolué pas seulement au niveau physique mais aussi spirituel et moral et le singe est resté à un niveau bien inférieur c'est-à-dire très laid.

\subsection{Temps}

Le temps est encore une notion présente dans toutes les langues puisque c'est ce qui nous détermine tous. II est notamment intéressant comment on perçoit le terme " jamais ". C'est un terme avec lequel on aime toujours lier une métaphore ou une image pour diminuer l'effet de quelque chose qui ne va jamais arriver ou se passer. Des expressions signifient depuis toujours : 
Le point de départ est souvent un événement de la mythologie ou de la Bible qui nous paraît très ancien. Ex. "Quand le Dieu marchait sur la Terre ».

Quand quelqu'un commence à nous raconter quelque chose et à s'étendre longuement sur un sujet on dit en BCMS : od Kulina bana - depuis ban Kulin. Equivalent en français - remonter au déluge; raconter $a b$ ovo. II est certain que ban Kulin était le ban de Bosnie du XII ${ }^{\mathrm{e}}$ siècle. Pour expliquer cette sorte d'expression comme repère du temps on utilise toujours un évènement très important du passé. Alors on y compte : les déluges - ce qui était le cas en français ; le tremblement de terre ; la famine ; les maladies ; les guerres dévastatrices etc. Puisque ces évènements ont eu un effet fort sur la civilisation, on s'en souvient et on en fait des mythes d'autant plus que l'homme ne peut pas les expliquer.

Dans l'expression od Kulina bana (depuis le temps de ban Kulin) il est intéressant de savoir que pendant la période de la gouvernance de ban Kulin, la Bosnie a connu l'âge d'or. Les gens avaient de tout en abondance. Mais les gens de Bosnie ont connu la catastrophe après la chute de ban Kulin d'où vient le parallèle avec le déluge et les autres catastrophes dont on se souvient d'une civilisation, ce qui reste un support utile pour les expressions.

Pour désigner le temps, nous avons aussi les expressions en BCMS telles que : kad na vrbi rodi grožđe " quand on recueillera le raisin du saule »; na kukovo ljeto, na Đurin petak, na svetog Živka "à la saint Živko » qui en effet n'existe pas dans le calendrier de l'église.

Remettre aux calendes grecques est l'expression traduite du latin (ad calendas graecas) employé par Auguste, selon Suétone, à propos des débiteurs insolvables. Elle contient une contradiction interne qui ne pouvait être sensible qu'aux Romains. Calendae, en latin, désigne le premier jour de chaque mois et bien évidemment, ce terme n'avait pas cours dans la Grèce antique. Cette plaisanterie savante est entrée dans l'usage commun, sans que l'expression de l'impossibilité soit située dans l'incompatibilité sémantique entre le nom et l'adjectif. En français courant c'est le mot calendes à lui seul qui emporte cette idée.

Chez les Serbes, le jour de saint George était le jour déterminé pour le paiement des impôts et d'autres taxes proclamé par l'assemblée nationale en 1815 en Serbie. Depuis que ce jour a perdu cette fonction et que le début de l'année est le $1^{\text {er }}$ janvier, l'expression $O$ Đurđevu dne (au jour de saint George) signifie « jamais ».

Pour dire " jamais " dans la phraséologie serbe, on utilise souvent les arbres qui auront des fruits qu'ils n'ont pas normalement. Ex : quand sur l'érable on aura les poires. En français on a les expressions suivantes : quand les poules auront des dents, la semaine des quatre jeudis, à la saint-glinglin. L'idée est toujours de faire la phrase la plus drôle possible afin de choquer l'interlocuteur. II s'agit souvent des expressions transparentes parce qu'on conçoit tout de suite qu'il s'agit d'un jour qui n'arrivera jamais. 
Puisqu'on sait qu'en roumain il y a aussi une expression telle que : quand les poires tomberont des peupliers, on conclut qu'il s'agit d'un modèle universel. Il s'agit en effet de la représentation universelle du paradis. Quel arbre choisir dépend de chaque langue individuellement et surtout de la rime, de l'assonance ou de l'allitération. C'est pourquoi on dit en BCMS : kad na vrbi zasvrbi « quand sur le saule ça démangera ». Une autre explication pour l'expression qu'un arbre donnera des fruits qu'il ne donne pas normalement est aussi d'utiliser un arbre qui n'est pas fertile pour les fruits et pour donner cette image bizarre on dit que cet arbre aura des fruits comme les pommes, les poires ou autre. Donc, le jour où le saule aura du raisin ou autre fruit est réservé à un autre temps où on aura encore une fois le paradis et ce sera l'œuvre de Dieu. II s'ensuit que ce temps pour le moment est « jamais » mais il ne faut pas perdre l'espoir, peut-être un jour verra-t-on encore une fois ce jardin de paradis.

\section{Conclusion}

Quand on parle d'origine des expressions BCMS, nous nous fondons plutôt sur la culture des langues BCMS, et bien qu'il y ait parfois une courte explication d'une expression dont l'utilisation est présente en bulgare ou en macédonien, nous ne pouvons pas dire que nous traitons les expressions autres que celles des langues BCMS. Pour ce qui est de la mythologie et des croyances, nous avons parfois mentionné la culture commune des slaves dont chaque langue a pris l'héritage.

Finalement les expressions sont souvent basées sur l'expérience quotidienne qui varie peu d'un pays à l'autre car cette expérience est commune à I'homme en général ou en tout cas à l'intérieur d'une même culture. D'anciens modes de vie dans les deux cultures nous ont légué des expressions figées dans une forme et dans un sens désuets, qui ont la vie d'autant plus durable qu'on en comprend moins la signification première, ce qui est, on l'a dit la principale cause de succès pour une expression.

\section{Références bibliographiques}

Belić, Aleksandar. Oko našeg književnog jezika [Autour de notre langue littéraire]. Beograd: Zavod za udžbenike, 1951.

Duneton, Claude. La Puce à l'oreille. Paris : Le livre de poche, 1990.

Čajkanović, Veselin. O magiji i religiji [De la magie et de la religion]. Belgrade : Biblioteka baština, 1985.

Čajkanović, Veselin. Stara srpska religija i mitologija [Ancienne religion et mythologie serbe]. Belgrade : Srpska akademija nauka i umjetnosti, Srpska književna zadruga, 1955. 
Drašković, Vlado. Francusko-srpskohrvatski rječnik sa poslovicama. [Dictionnaire français serbo-croate avec les proverbes]. Belgrade : Zavod za udžbenike i nastavna sredstva, 1990.

Gross, Gaston. Les expressions figées en français. Noms composés et autres locutions. Paris : Ophrys, 1996.

Guiraud, Pierre. Les locutions françaises. Paris : Presses Universitaires de France, 1973.

Le Petit Robert, Paris : Dictionnaires Le Robert, 2004.

Littré, Emile. Dictionnaire de la Langue française. Paris : Gallimard, 1959.

Matešić, Josip. Frazeološki rječnik hrvatskoga ili srpskoga jezika [Dictionnaire phraséologique du croate ou du serbe], Zagreb, Školska knjiga, 1982.

Milosavljević, Boško. Srpsko-francuski rječnik idioma i izreka. [Dictionnaire des idiomes et des dictons serbe-français]. Belgrade : Srpska književna zadruga, 1994.

Mrazovac Pavica et Primorac Ružica. Nemačko-srpskohrvatski frazeološki rečnik.

[Dictionnaire phraséologique allemand-serbo-croate]. Beograd, 1981.

Mršević-Radović, Dragana. Frazeologija i nacionalna kultura [Phraséologie et culture nationale], Beograd, Biblioteka književnost i jezi, 2008.

Rey, Alain et Chantreau, Sophie. Dictionnaire d'expressions et locutions. Paris : Le Robert, 2003.

Šipka, Milan. Zašto se kaže ? [Pourquoi dit-on ? ]. Novi Sad : Prometej, 2008.

Stefanović-Karadžić, Vuk. Srpski rječnik. [Dictionnaire de la langue serbe]. Beograd: Zavod za udžbenike, 1856.

Zouogbo, Jean-Philippe Claver. Le proverbe entre langues et cultures : une étude de linguistique confrontative allemand / français / bété. (= Études contrastives, vol. 10). Francfort/Main : Peter Lang, 2009.

\section{ORIGINS OF IDIOMS}

Many idiomatic expressions, in their original use, were not figurative but had literal meaning. Also, sometimes the attribution of a literal meaning can change as the phrase becomes disconnected from its original roots, leading to a folk etymology. However, at the beginning all the have been motivated: comparison, metaphor, images or clichés, some have completely lost their first meaning, others are still addressed to the speaker's imagination and suggest a relationship with their etymology.

Key words: origin, etymology, expression, translation. 